\title{
Linfadenitis cervical por Salmonella no Typhi en una paciente diabética
}

\author{
M. Adelaida Velásquez, Juliana Ortiz, Santiago Atehortúa y Carlos A. Agudelo
}

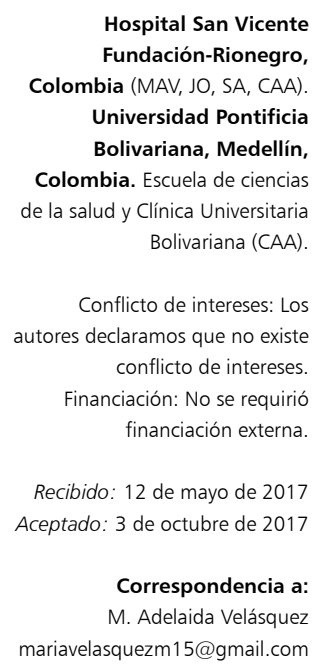

Hospital San Vicente Fundación-Rionegro, Colombia (MAV, JO, SA, CAA). Universidad Pontificia Bolivariana, Medellín, Colombia. Escuela de ciencias de la salud y Clínica Universitaria Bolivariana (CAA)

Conflicto de intereses: Los autores declaramos que no existe conflicto de intereses. Financiación: No se requirió financiación externa.

Recibido: 12 de mayo de 2017 Aceptado: 3 de octubre de 2017

Correspondencia a: M. Adelaida Velásquez mariavelasquezm15@gmail.com

\section{Introducción}

L a infección por Salmonella no Typhi es una de las enfermedades transmitidas por alimentos más común y ampliamente extendida. Se estima que ocurren más de 93 millones de casos de gastroenteritis debido a Salmonella spp. y más de 155.000 muertes alrededor del mundo ${ }^{1}$. Salmonella es un género bacteriano que pertenece a la familia Enterobacteriaceae. Es un bacilo gramnegativo, anaerobio facultativo, de carácter zoonótico y distribución universal. Se transmite por contacto directo o contaminación cruzada durante la manipulación en el procesamiento de alimentos o en el hogar; pero también se ha descrito transmisión por vía sexual. Aunque la mayoría de los casos causa un cuadro gastrointestinal, hasta $5 \%$ ocasionan bacteriemia y de éstos cerca de $40 \%$ producen infecciones focales extraintestinales ${ }^{2,3}$. Estas manifestaciones extraintestinales pueden afectar prácticamente cualquier órgano, pero ocurren principalmente en hueso, articulaciones, sistema nervioso central y tejidos blandos ${ }^{4}$. La adenitis, como manifestación aislada, es una forma inusual de presentación de la enfermedad.

Comunicamos el caso clínico de una adenitis como única manifestación de una infección por Salmonella no Typhi en una paciente con diabetes mellitus de reciente diagnóstico.

\section{Caso clínico}

Mujer de 67 años de edad, sin antecedentes patológicos conocidos, residente en una zona rural del municipio de Segovia, Antioquia, Colombia. Consultó por la aparición de una masa cervical de un mes de evolución, de creci- miento progresivo, acompañada de fiebre, escalofríos y síntomas inflamatorios locales, sin síntomas gastrointestinales y sin información epidemiológica relevante. Al examen físico se palpó una masa fluctuante, acompañada de eritema y calor, en la región submandibular izquierda.

En los exámenes de laboratorio iniciales se encontró una hemoglobina de $11 \mathrm{~g} / \mathrm{dl}$, leucocitos de 14.200 cél/ $\mathrm{mm}^{3}$, neutrófilos de $73 \%$ y plaquetas de $622.000 / \mathrm{mm}^{3}$. La proteína $\mathrm{C}$ reactiva inicial fue de $1,25 \mathrm{mg} / \mathrm{dl}(\mathrm{VN}$ $<0,82 \mathrm{mg} / \mathrm{dl}$ ), la deshidrogenasa láctica de $217 \mathrm{U} / \mathrm{L}$ (VN 125-243 U/L). Una prueba de inmunoensayo de cuarta generación para VIH fue negativa. Los valores de la aspartato aminotransferasa, la alanino aminotransferasa, la bilirrubina total y la creatinina fueron normales. Un resultado de hemoglobina glicosilada de $8,3 \%$ hizo el diagnóstico de diabetes mellitus, no conocido con anterioridad en la paciente.

La tomografía computarizada (TC) cervical mostró una lesión única de 55 x 47 x 90 mm, mal definida, con una zona central de baja densidad, centro necrótico, abscedada, que comprometía los planos musculares del hemicuello izquierdo (Figura 1). Por sospecha de una lesión neoplásica, la paciente fue llevada a cirugía para la extracción de la masa. En el acto operatorio se encontró una lesión abscedada que drenó abundante pus. El examen histológico reveló la presencia generalizada de necrosis de licuefacción y pérdida completa de la estructura del órgano. En el post-operatorio inmediato se inició tratamiento antibacteriano empírico con piperacilina/tazobactam y vancomicina. Los cultivos de secreción para aerobios en medios tradicionales y la identificación por el sistema VITEK2 ${ }^{\circledR}$ (bioMérieux ${ }^{\circledR}$ ) permitieron el aislamiento y la identificación de Salmonella no Typhi sensible a fluoroquinolonas. No se contó con técnicas 
de serotipificación para la identificación del serovar. El esquema antimicrobiano empírico fue reemplazado por ciprofloxacina, la que se administró por un total de 14 días. La ecocardiografía transtorácica y la TC contrastada de abdomen fueron normales. La ecografía de la vesícula y las vías biliares reveló la presencia de una colelitiasis. La paciente se encontraba asintomática por lo que se realizó una colecistectomía electiva previa al alta, con el fin de controlar un posible reservorio. No se obtuvo cultivo de bilis. La paciente no refirió un cuadro gastrointestinal previo, ni contacto con personas enfermas o animales. Tuvo una evolución satisfactoria sin recidiva del cuadro clínico.

\section{Discusión}

La fiebre tifoidea, causada por Salmonella Typhi, fue la manifestación más importante de las infecciones por Salmonella spp. hasta la aparición de los antibacterianos. Desde entonces, las infecciones han sido provocadas en su mayoría por Salmonella no Typhi, siendo la infección gastrointestinal la manifestación predominante ${ }^{1,2}$. Aunque la mayoría de estas infecciones son leves, hasta $5 \%$ de los pacientes, principalmente aquellos inmunocomprometidos, pueden presentar un episodio de bacteriemia y hacer siembras extraintestinales ${ }^{1}$.

Prácticamente cualquier sitio anatómico puede ser afectado por una siembra hematógena y desarrollar una infección local, incluso después del tratamiento adecuado de una bacteriemia ${ }^{3}$. Aunque la localización extraintestinal en órganos como hueso, articulaciones, sistema nervioso central y tejidos blandos, es ampliamente conocida, el compromiso focal único de una cadena ganglionar es un hallazgo que ha sido pocas veces descrito en pacientes adultos ${ }^{2,3,5,6}$.

En una revisión de la literatura científica se encontraron 26 casos publicados de adenitis por Salmonella no Typhi. La mayoría de estos casos fueron en menores de edad, afectando principalmente las cadenas ganglionares mesentéricas y siendo inicialmente confundidos con cuadros de apendicitis aguda, a pesar de la presentación insidiosa de algunos de ellos ${ }^{7-11,21}$. Otros lugares afectados por Salmonella no Typhi en niños son las cadenas ganglionares de cabeza y cuello $^{5,12}$. Aunque en uno de los casos de adenitis por Salmonella no Typhi se describió una deficiencia del receptor $\beta 1$ de la IL 12/-23 como inmunosupresión asociada $^{5}$, los demás casos fueron comunicados en niños inmunocompetentes ${ }^{7,9,10,12}$.

En la revisión de la literatura realizada se encontraron casos de adenitis aislada por Salmonella no Typhi en adultos, cinco de ellos cervicales ${ }^{2,6,11,13,14}$. En todos los casos de adenitis cervical en adultos se describió una condición inmunosupresora de base, en dos se desarro-

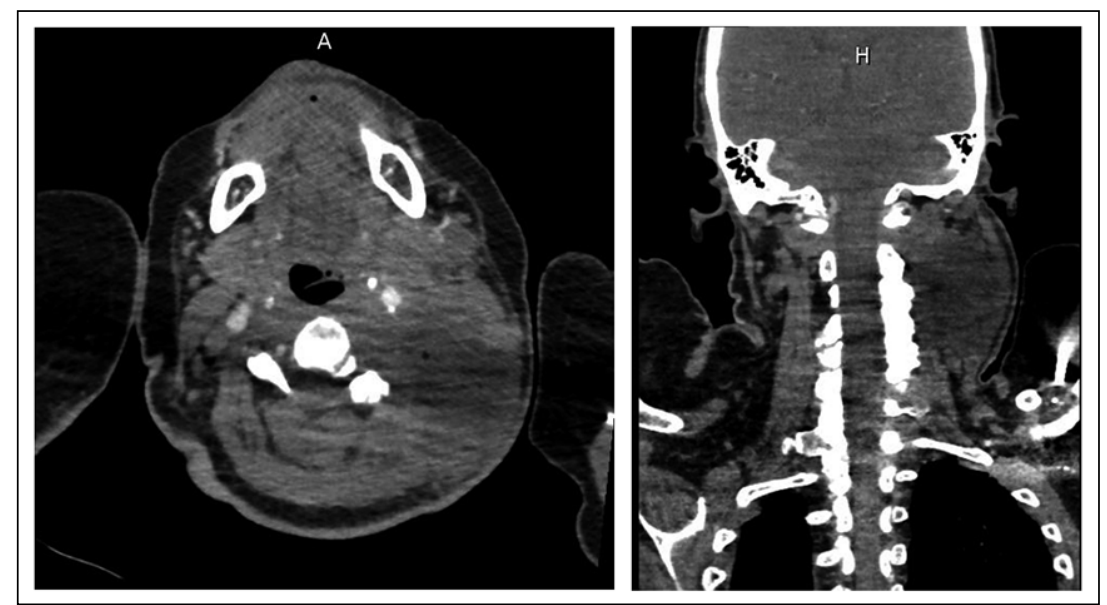

Figura 1. Tomografía computarizada (TC) corte axial y coronal: lesión única de 55 × 47 mm, mal definida, con una zona central de baja intensidad, que comprometía los planos musculares del hemicuello izquierdo.

llaron concomitante con un linfoma, otros dos pacientes tenían diabetes mellitus y otro, una sobrecarga de hierro secundaria a una $\beta$-talasemia ${ }^{2,6,13,14}$.

El compromiso cervical por Salmonella spp. puede presentarse también en forma de abscesos submandibulares o retrofaríngeos ${ }^{15-17}$. A diferencia del origen hematógeno o linfático de las adenitis cervicales, estos abscesos se producen por inoculación directa luego de la ingesta de Salmonella $\mathrm{spp}^{16}$. En nuestro caso no se pudo encontrar una asociación con un algún alimento de riesgo, síntomas gastrointestinales previos o contacto con personas enfermas o animales.

Varias investigaciones han demostrado un mayor riesgo de infecciones extraintestinales por Salmonella spp. en personas con diabetes mellitus ${ }^{6,18}$. Esta predisposición parece ser explicada por el aumento del $\mathrm{pH}$ gástrico, así como por el retraso en el vaciamiento gástrico y la disminución de la motilidad intestinal secundarios a la neuropatía autonómica, más que por las anormalidades en la función de los linfocitos T y los neutrófilos descritas para estos pacientes $2,6,13,18-20$.

\section{Conclusión}

Este caso clínico apoya la predisposición para las infecciones extraintestinales por Salmonella no Typhi en los pacientes diabéticos, principalmente si las cifras de glicemia no están controladas. Igualmente resalta la importancia de los estudios microbiológicos en pacientes inmunocomprometidos con compromiso de los ganglios linfáticos, aún en aquellos con alta sospecha clínica de cáncer. 


\section{Resumen}

La infección por Salmonella no Typhi es una de las enfermedades transmitidas por alimentos más común y ampliamente extendida en el mundo. Aunque la mayoría de los casos se limitan al tracto gastrointestinal, el compromiso ex- traintestinal no es infrecuente. Sin embargo, la adenitis como manifestación aislada, es una forma inusual de presentación de la enfermedad. Comunicamos el caso clínico de una mujer de 67 años de edad con diagnóstico de diabetes mellitus y una linfadenitis cervical por Salmonella no Typhi tratada con ciprofloxacina y y que requirió resección quirúrgica.

\section{Referencias bibliográficas}

1.- Sacristán-Rodríguez P, Pérez-Osorio L, Méndez-Rodríguez I. Prevalencia y susceptibilidad antimicrobiana de Salmonella spp. aislada de animales exóticos que conviven con niños. Medicina \& Labotarorio 2014; 20 : 169-84.

2.- Chen P L, Chang C M, Wu C J, Ko N Y, Lee N $\mathrm{Y}$, Lee H C, et al. Extraintestinal focal infections in adults with nontyphoid Salmonella bacteraemia: predisposing factors and clinical outcome. J Intern Med 2007; 261: 91-100.

3.- Pegues D A, Miller S I. Salmonella species. En: Bennett J E, Dolin R, Blaser M J, eds. Mandell, Douglas, and Bennett's Principles and Practice of Infectious Diseases, 8th ed. Philadelphia: Churchill Livingstone Elsevier, 2015; p 2567.

4.- Guarga A, Urrutia A, Paret A, Rey-Joly C. Mesenteric lymphadenitis caused by Salmonella enteritidis. Med Clin (Barc) 1984; 82: 136.

5.- van de Vosse E, Ottenhoff T H, de Paus R A, Verhard E M, de Boer T, van Dissel J T, et al. Mycobacterium bovis BCG-itis and cervical lymphadenitis due to Salmonella enteritidis in a patient with complete interleukin-12/-23 receptor beta1 deficiency. Infection 2010; 38: 128-30.

6.- Dodd E, Mitchell J, Kearney S, Sidman J.
Salmonella lymphadenitis mimicking neoplastic process in a teen. Minn Med 2015; 98: 38.

7.- García-Corbeira P, Ramos J M, Aguado J M, Soriano F. Six cases in which mesenteric lymphadenitis due to non-typhi Salmonella caused an appendicitis-like syndrome. Clin Infect Dis 1995; 21: 231-2.

8.- Martin H C, Goon H K. Salmonella ileocecal lymphadenitis masquerading as appendicitis. J Pediatr Surg 1986; 21: 377-8.

9.- $\quad$ Arda I S, Ergin F, Varan B, Dermirhan B, Aslan H, Ozyaylali I. Acute abdomen caused by Salmonella typhimurium infection in children. J Pediatr Surg 2001; 36: 1849-52.

10.- Mautner L S, McIntyre A J, Ord J V. Isolation of Salmonella organisms from mesenteric lymph nodes. Can Med Assoc J 1961; 85: 13941.

11.- Lee J H, Rhee P L, Lee J K, Lee K T, Son $\mathrm{H}$ J, Kim J J, et al. The etiology and clinical characteristics of mesenteric adenitis in Korean adults. J Korean Med Sci 1997; 12: 105-10.

12.- Murray J C, Singh R R, Brandt M L, Kearney D L, Ogden A K. Granulomatous submandibular lymphadenitis caused by Salmonella species in a healthy child. Clin Infect Dis 1994; 19: 11756.

13.- Pastagia M, Jenkins S G. Salmonella neck abscess as an opportunistic infection in diabetes mellitus. Case Rep Infect Dis 2013; 2013: 708419.
14.- Campbell W N. Salmonella lymphadenitis associated with undiagnosed lymphoma. Eur J Clin Microbiol Infect Dis 2001; 20: 359-61.

15.- Su F H, Chen P T, Chiu Y C, Chen J W. Salmonella retropharyngeal abscess in a child: case report and literature review. Pediatr Infect Dis J 2003; 22: 833-6.

16.- Bahar G, Dansuk Z, Kocatürk S, Cakir T, Mert A. Abscess of the neck caused by Salmonella enteritidis. Otolaryngol Head Neck Surg 2003; 129: 445-7.

17.- Behr M A, McDonald J. Salmonella neck abscess in a patient with beta-thalassemia major: case report and review. Clin Infect Dis 1996; 23: 404-5.

18.- Margaret A P, Solomon P J, Lohith H. A rare case of typhoid presenting with fever, ascites, hyponatremia, thrombocitopenia, mesenteric lymphadenitis and multi-drug resistance. $\mathrm{J}$ Pharm Bioallied Sci 2015; 7 (Suppl 1): S65-6.

19.- Telzak E E, Greenberg M S, Budnick L D, Singh T, Blum S. Diabetes mellitus- a newly described risk factor for infection from Salmonella enteritidis. J Infect Dis 1991; 164 538-41.

20.- Hohmann E L. Nontyphoidal salmonellosis. Clin Infect Dis 2001; 32: 263-9.

21.- Likitnukul S, Wongsawat J, Nunthapisud P. Appendicitis-like syndrome owing to mesenteric adenitis caused by Salmonella typhi. Ann Trop Pediatr 2002; 22: 97-9. 